\title{
NONLINEAR DYNAMIC RESPONSES OF ARCH DAM WITH SHEAR KEYS
}

\author{
Chengbin Du, Shouyan Jiang \\ Mechanics and Materials Experiment Center, Hohai University, 210098 \\ Nanjing, Jiangsu, P. R. China, cbdu@hhu.edu.cn
}

\begin{abstract}
The purpose of this paper is to obtain an insight into the effects of shear keys with different slopes on the nonlinear seismic responses of an arch dam. The nonlinear exponential dynamic contact constitutive model is proposed for simulating the normal interactions of the two surfaces separated by contraction joints, along with the standard Coulomb friction model to simulate the tangential interactions. After the shear key's real configuration is established, the seismic responses of the arch dam are discussed in detail to understand the effects of shear keys on the behavior of the contraction joints and the stress and deformation of the dam. The results indicate that the dam without the shear keys shows comparatively narrow joint opening, and that the maximum joint opening decreases when the slope of shear keys increases. In addition, the slope of shear keys exerts an obvious effect on the sliding displacement along the radial direction and the stress of the dam.
\end{abstract}

Keywords- Dynamic Responses, Arch Dam, Contraction Joints, Shear Keys

\section{INTRODUCTION}

Taken into consideration the release of thermal stress in mass concrete coupling with the improvement of dam integrity, arch dams are built as assemblages of monoliths separated by contraction joints with shear keys. However, the relative motion may occur between adjacent monoliths under earthquake-induced medium-intensity ground motion, resulting in the opening and closing of the joints and the possible shear movement at the joint locations [1]. In the past two decades, several joint models have been proposed to simulate the motion of contraction joints by other researchers [2-4], but their studies rarely took into account the real configuration of shear keys. Hohberg [5] approximately simulates the effects of shear keys by increasing the height of the joint asperity. Lau et al. [6] and Zhang et al. [7] both introduced linear elastic springs to simulate the effects of shear keys on the joints. In fact, the force-transfer capability of shear keys is relative to the joint opening. Therefore, the real configuration of shear keys can more realistically reflect the capability of shear keys. It is thereby analyzed in detail in this paper that the effects of shear keys with different slopes on the nonlinear seismic responses of an arch dam, which is on the basis of the real configuration of shear keys that established in finite element models.

\section{DYNAMIC CONTACT CONSTITUTIVE MODEL}

The method for imposing the contact condition is discussed in this section. The node-to-surface contact discretization is one of the most commonly used methods in the finite element method (FEM) of contact problems and is used in this paper. 


\subsection{Contact Kinematic Variables}

As shown in Fig. 1, at the (i-1)th iteration of time $t+\Delta t$, the slave node $P$ with coordinates ${ }^{t+\Delta t} \mathbf{x}_{P}^{(i-1)}$ comes into contact with the master surface $A-B-C-D$ defined by the nodal coordinates ${ }^{t+\Delta t} \mathbf{x}_{A}^{(i-1)},{ }^{t+\Delta t} \mathbf{x}_{B}^{(i-1)}$, ${ }^{t+\Delta t} \mathbf{x}_{C}^{(i-1)}$ and ${ }^{t+\Delta t} \mathbf{x}_{D}^{(i-1)}$. Point $Q$ on the master surface $A$ $B-C-D$ is defined to be the physical point of contact of node $P$. By introducing the local coordinate $\xi \eta$ in the master surface, the coordinate of the point $Q$ can be obtained by

$$
{ }^{t+\Delta t} \mathbf{x}_{Q}^{(i-1)}=\sum_{i=1}^{4} N_{i}(\xi, \eta)^{t+\Delta t} \mathbf{x}_{i}^{(i-1)}, \quad i=A, B, C, D
$$

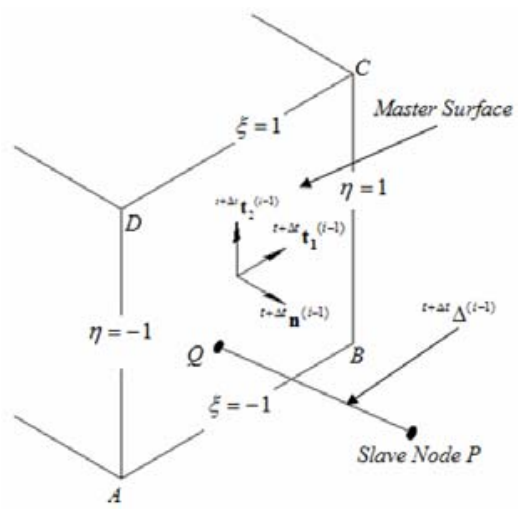

Fig.1 Node-to-surface contact

where, $N_{i}$ is the shape function of the standard FEM with 4-node isoparametric element. The overlap ${ }^{t+\Delta t} \Delta^{(i-1)}$ between the slave node $P$ and the point $Q$ is thereby obtained by

$$
{ }^{t+\Delta t} \Delta^{(i-1)}={ }^{t+\Delta t} \mathbf{x}_{Q}^{(i-1)}-{ }^{t+\Delta t} \mathbf{x}_{P}^{(i-1)}
$$

The normal and tangential components of ${ }^{t+\Delta t} \boldsymbol{\Delta}^{(i-1)}$ can be expressed as

$$
{ }^{t+\Delta t} \Delta_{N}^{(i-1)}={ }^{t+\Delta t} \mathbf{n}^{(i-1)} \cdot{ }^{t+\Delta t} \Delta^{(i-1)},{ }^{t+\Delta t} \Delta_{1}^{(i-1)}={ }^{t+\Delta t} \mathbf{t}_{1}{ }^{(i-1)} \cdot{ }^{t+\Delta t} \Delta^{(i-1)},{ }^{t+\Delta t} \Delta_{2}^{(i-1)}={ }^{t+\Delta t} \mathbf{t}_{2}{ }^{(i-1)} \bullet^{t+\Delta t} \Delta^{(i-1)}
$$

where, ${ }^{t+\Delta t} \Delta_{N}^{(i-1)}$ is the overlap between the node $P$ and the point $Q$ in normal direction ${ }^{t+\Delta t} \mathbf{n}^{(i-1)}$, which can represent joint opening if ${ }^{t+\Delta t} \Delta_{N}^{(i-1)} \leq-\varepsilon$ where $\varepsilon$ is the overlap tolerance that is set to infinitesimal; ${ }^{t+\Delta t} \Delta_{1}^{(i-1)}$ denotes the sliding displacement along slip direction ${ }^{t+\Delta t} \mathbf{t}_{1}^{(i-1)}$, and ${ }^{t+\Delta t} \Delta_{2}^{(i-1)}$ along slip direction ${ }^{t+\Delta t} \mathbf{t}_{2}^{(i-1)} \cdot{ }^{t+\Delta t} \Delta_{N}^{(i-1)}>-\varepsilon$ means contact occurs with a contact force ${ }^{t+\Delta t} \lambda^{(i-1)}$ at node $P$. For the master surface $A-B-C-D$, the equivalent contact forces at node $A, B, C$ and $D$ can be expressed as follows.

$$
{ }^{t+\Delta t} \lambda_{i}^{(i-1)}=-N_{i}{ }^{t+\Delta t} \lambda^{(i-1)}, \quad i=A, B, C, D
$$

${ }^{t+\Delta t} \lambda_{N}^{(i-1)}$ is the normal contact force defined as ${ }^{t+\Delta t} \lambda_{N}^{i-1}={ }^{t+\Delta t} \mathbf{n}^{(i-1)}{ }^{t+\Delta t} \lambda^{(i-1)}$, the tangential contact force as ${ }^{t+\Delta t} \lambda_{i}^{i-1}={ }^{t+\Delta t} \mathbf{t}_{i}{ }^{(i-1)} \bullet^{t+\Delta t} \lambda^{(i-1)}, i=1,2$.

\subsection{Normal Contact Model}

The soft contact method [8] is adopted for simulating the normal interactions. The following nonlinear exponential constitutive relation is implemented in the contact boundary to prevent the slave nodes from penetrating into the master surface. It should be noted that the superscripts in Eq. (5) as well as the equations in section 2.3 and 2.4 are omitted, because the relations is suitable for the whole solving processes.

$$
\begin{cases}\lambda_{N}=0, & \text { if } \quad \Delta_{N} \leq-g_{0} \\ \lambda_{N}=\frac{\lambda_{0}}{e-1}\left[\left(\frac{\Delta_{N}}{g_{0}}+1\right)\left(e^{\frac{\Delta_{N}}{g_{0}}+1}-1\right)\right], & \text { if } \Delta_{N}>-g_{0}\end{cases}
$$

where, $\lambda_{0}$ and $g_{0}$ are selected parameters; $\lambda_{0}$ is the critical normal contact force at zero overlap; $g_{0}$ is the normal gap at zero normal contact force. The relation shown in Eq.(5) 
can make the normal contact force varies smoothly and is beneficial to improve the process of converge. The overlap tolerance $\varepsilon$ mentioned in section 2.1 is equal to $g_{0}$.

\subsection{Tangential Contact Model}

The relationship between the elastic slip and the interface shear force satisfies the equation

$$
\lambda_{i}=k_{s} \Delta_{i}^{e l} \quad(i=1,2)
$$

where, $\Delta_{i}^{e l}$ is the elastic slip at the end of the current iteration that is reversible and $k_{s}=\lambda_{\text {crit }} / \Delta_{\text {crit }}$ is called the stiffness in elastic stick, and $\Delta_{\text {crit }}$ is the allowable maximum elastic slip, which is set to be $0.5 \%$ of the average length of all contact elements in the model. For the elastic stick status, the elastic slip is equal to the total slip.

When $\lambda_{e q}=\mu \lambda_{N}$, relative motion between the two contact surfaces occurs. Suppose that the starting situation at the current iteration is characterized by the elastic slip $\tilde{\Delta}_{i}^{e l}$, the elastic slip at the end of the iteration is $\Delta_{i}^{e l}$, and the plastic slip increment is $\Delta_{i}^{p l}$. Thus, the total slip increment at the current increment can be expressed as

$$
\Delta_{i}=\Delta_{i}^{e l}-\tilde{\Delta}_{i}^{e l}+\Delta_{i}^{p l} \quad(i=1,2)
$$

At the end of the current iteration, the shear force is

$$
\lambda_{i}=k_{s} \Delta_{i}^{e l}=\frac{\lambda_{\text {crit }}}{\Delta_{\text {crit }}} \Delta_{i}^{e l} \quad(i=1,2)
$$

and the relationship, between the plastic slip increment and the shear force is

$$
\Delta_{i}^{p l}=\frac{\lambda_{i}}{\lambda_{\text {crit }}} \Delta_{e q}^{p l} \quad(i=1,2)
$$

Substituting Eq. (8) and Eq. (9) into Eq. (7), the following equation can be obtained

$$
\lambda_{i}=\frac{\tilde{\Delta}_{i}^{e l}+\Delta_{i}}{\Delta_{\text {crit }}+\Delta_{\text {eq }}^{\text {pl }}} \lambda_{\text {crit }} \quad(i=1,2)
$$

Let $\Delta_{i}^{p r}=\tilde{\Delta}_{i}^{e l}+\Delta_{i}$, Eq. (10) can be simplified as

$$
\lambda_{i}=\frac{\Delta_{i}^{p r}}{\Delta_{\text {crit }}+\Delta_{\text {eq }}^{p l}} \lambda_{\text {crit }} \quad(i=1,2)
$$

Substituting $\Delta_{e q}^{p l}=\Delta_{e q}^{p r}-\Delta_{c r i t}$, where $\Delta_{e q}^{p r}=\sqrt{\left(\Delta_{1}^{p r}\right)^{2}+\left(\Delta_{2}^{p r}\right)^{2}}$, into Eq. (11), we can obtain

$$
\lambda_{i}=n_{i} \lambda_{\text {crit }} \quad(i=1,2)
$$

where, $n_{i}=\lambda_{i}^{p r} / \lambda_{e q}^{p r}$.

\section{NONLINEAR DYNAMIC RESPONSES OF AN ARCH DAM}

\subsection{Numerical Model of an Arch Dam}

The real configurations of the dam contain two structural joints with trapezoidal shear keys. The gravity arch dam is $99 \mathrm{~m}$ high, $6.0 \mathrm{~m}$ wide at the dam crest and $42.0 \mathrm{~m}$ wide at the bottom of arch crown. The elevation of the dam base is $1004.00 \mathrm{~m}$. The outer radius of the arch ring for the dam is $148 \mathrm{~m}$. As shown in Fig.2, the dam and the foundation both are discretized into hexahedron elements, with 14142 elements and 
17744 nodes. The simplified trapezoidal shear key, using a big trapezoidal shear key to represent all small trapezoidal shear keys, is shown in Fig.2(c).

In the analysis, the mass density of concrete is taken as $2400 \mathrm{~kg} / \mathrm{m}^{3}$, the elastic modulus as $22 \mathrm{GPa}$ at elevations lower than $1039 \mathrm{~m}$, and at elevations higher than 1039 $\mathrm{m}$ the elastic modulus is $25.5 \mathrm{GPa}$, and Poisson ratio is 0.167 . For the foundation material, the elastic modulus is $20 \mathrm{GPa}$, and the Poisson's ratio is 0.30 . Damping of the system is assumed to be of the Rayleigh type with 5\% damping in the first and second vibration modes of the dam with a full reservoir. The selected parameters in Eq.(5) are $\lambda_{0}=5 \times 10^{9}$ and $g_{0}=5 \times 10^{-7}$. The dynamic friction coefficient is set to be 0.6 . The applied static loads include the weight of the dam itself, the hydrostatic pressure of the impounded water with a free surface of $96 \mathrm{~m}$ above the base and the uplift pressure. The dynamic excitations include $10 \mathrm{~s}$ of artificial earthquake with the peak acceleration 0.15 g. The dam-reservoir interaction is accounted for by the Westergaard added mass approach. In addition, a finite massless foundation model is used to include the damfoundation interaction. The Newmark method with a time step of $0.01 \mathrm{~s}$ is used for the integration of the equations of motion.

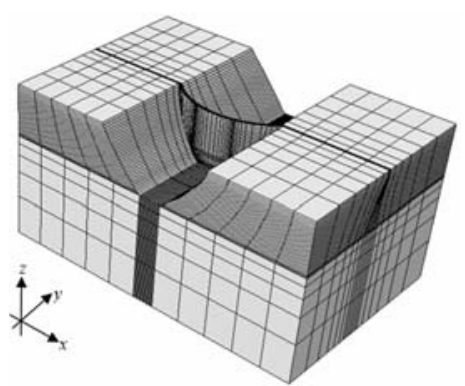

(a) FE mesh of dam-foundation

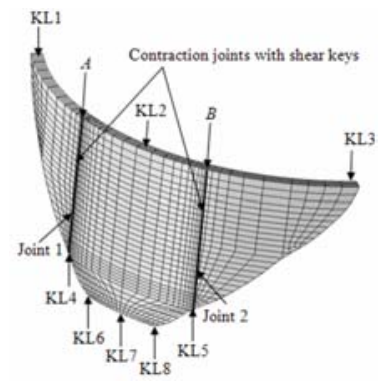

(b) FE mesh of dam

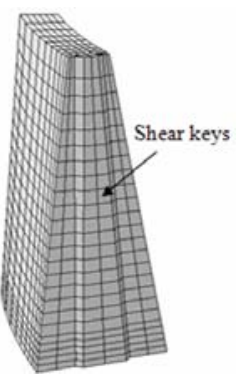

(c) Shear Keys

Fig.2 Numerical models of an arch dam

The slope $m$ of the shear keys is the main characteristic parameter of the shear keys. Herein, the main concerns are the effects of the slope $m$ of the shear keys on the dynamic responses of the arch dam. Six cases are studied to investigate the seismic response of the arch dam. These are summarised in Table 1.

Table 1 Cases for studying the effects of the slope $m$

\begin{tabular}{ccc}
\hline Case No. & Slope $m$ & Remarks \\
\hline Case 1 & 0 & Dam without shear keys \\
Case 2 & Infinity & Dam with rectangle shear keys \\
Case 3 & $3.732: 1$ & Dam with trapezoidal shear keys \\
Case 4 & $1.732: 1$ & Dam with trapezoidal shear keys \\
Case 5 & $1: 1$ & Dam with trapezoidal shear keys \\
Case 6 & $0.7: 1$ & Dam with trapezoidal shear keys \\
\hline
\end{tabular}

\subsection{Responses of Joint Opening and Sliding at the Contraction Joints}

The envelope of the maximum joint opening with elevation for the joint 2 is shown in Fig.3. Comparing the joint opening in case 1 with that in other cases, the 
results indicate that the slope of the shear keys has a great effect on the joint opening. The dam without shear keys shows a lower joint opening. It is thereby indicated that the plane joints model (case 1) cannot encompass the nonlinear seismic responses of arch dam. To model the nonlinear dynamic responses of arch dams more realistically, the effects of shear keys must be considered. Additionally, it is also found that the maximum joint opening decreases with increasing slope $m$ of shear keys. Time histories of the joint opening at point $A$ are plotted in Fig. 4.

The maximum sliding displacement of joints at point $A / B$ is shown in Table 2 . Compared with the dam without shear keys, the dam with shear keys can effectively restrain the sliding motion along the radial direction. The slope of shear keys also exerts an obvious effect on the sliding displacement along the radial direction. Comparison of the results of cases 3, 4, 5 and 6 shows that with a smaller slope, a larger sliding displacement along the radial direction develops. However, the results in cases 2 and 3 show a negligible difference.

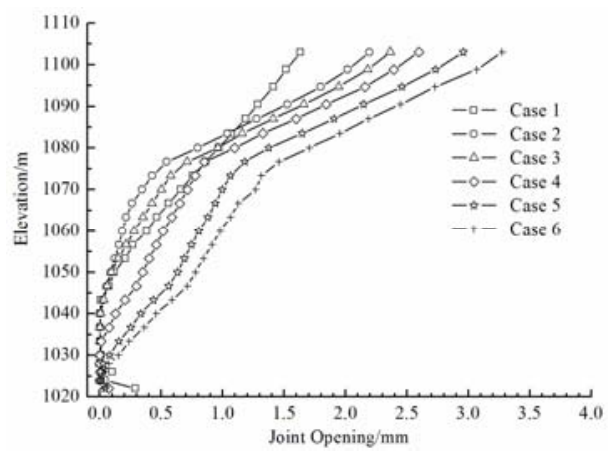

Fig. 3 Envelope of maximum joint opening with elevation for the joint 2

Table 2 Maximum sliding displacement $/ \mathrm{mm}$

\begin{tabular}{ccc}
\hline Locations & Point $A$ & Point $B$ \\
\hline Case 1 & 32.82 & 21.90 \\
Case 2 & 2.09 & 1.60 \\
Case 3 & 2.07 & 1.59 \\
Case 4 & 2.17 & 2.31 \\
Case 5 & 3.82 & 3.64 \\
Case 6 & 5.45 & 5.19 \\
\hline
\end{tabular}

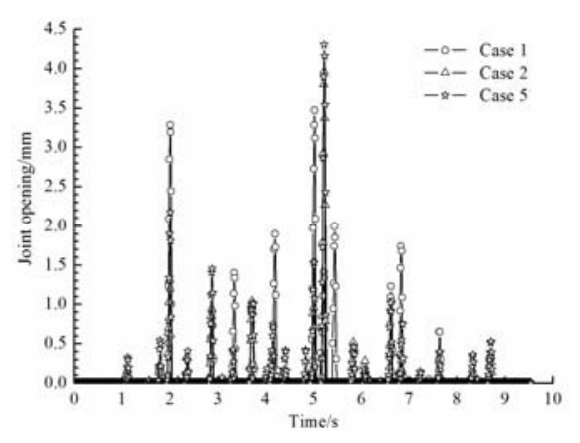

Fig. 4 Time histories of joint opening at point $A$

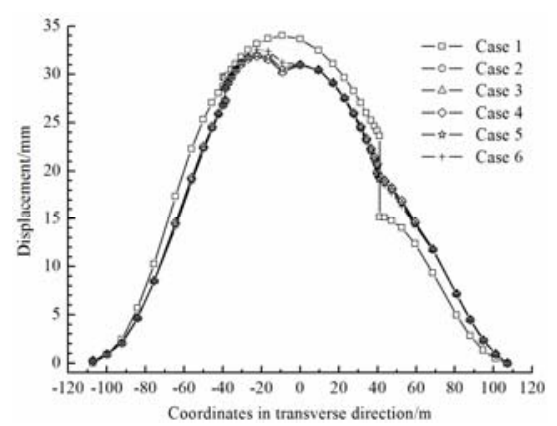

Fig. 5 Maximum stream displacements along dam crest

\subsection{Responses of Stress and Deformation for the Dam}

The maximum stream displacements along the dam crest are shown in Fig. 5. The displacement in the analysis in case 1 is larger than that in cases 2, 3, 4, 5 and 6. The phenomenon indicates that setting shear keys at the contraction joint locations is indeed beneficial to improve the integrity of arch dam. However, it can also be found that the displacements in cases 2, 3, 4, 5 and 6 have a slight difference.

The comparison of the results of the stress from cases 1 to 6 indicates that effects of the shear keys significantly decreased the principal tensile stress at the bottom 
of the contraction joints as well as at the bottom of the dam. However, at the dam crest, the shear keys increased the principal tensile stress greatly. Additionally, the results of cases 2 and 3 are slightly different. According to the results of cases 4, 5 and 6 , for the shear keys with a smaller slope $m$, the principal tensile stresses at the bottom of the contraction joints increase.

\section{CONCLUDING REMARKS}

In summary, some conclusions can be drawn: (1) the dam without shear keys showed a comparatively narrow joint opening. Therefore, the plane joints model could not encompass the nonlinear seismic responses of the arch dam. To model the nonlinear dynamic response of arch dams more realistically, the effects of shear keys must be considered. (2) Compared to the dam without shear keys, the dam with shear keys could effectively restrain the sliding motion along the radial direction. The slope of shear keys also had an evident effect on the sliding displacement along the radial direction. (3) The effects of shear keys decreased the principal tensile stress at the bottom of the contraction joints as well as at the bottom of the dam significantly.

\section{ACKNOWLEDGEMENTS}

The authors gratefully acknowledge support for this research from the National Basic Research Program of China (973 Program, Grant No. 2007CB714104), National Natural Science Foundation of China (Grant No.50779011) and Open Research Fund supported by IWHR (Grant No.2008538613) .

\section{REFERENCES}

1. A. Niwa and R. W. Clough, Nonlinear seismic response of arch dams, Earthquake Engineering and Structural Dynamics 10, 267-281, 1982.

2. M. L. Dowling and J. F. Hall, Nonlinear seismic analysis of arch dams, Journal of Engineering Mechanics 115, 768-789, 1989.

3. X. L. Du and J. Tu. Nonlinear seismic response analysis of arch dam-foundation systems- part II opening and closing contact joints, Bulletin of Earthquake Engineering 5, 121-133, 2007.

4. H. Arabshahi, V. Lotfi, Nonlinear dynamic analysis of arch dams with joint sliding mechanism, Engineering Computations 26, 464-482, 2009.

5. J. M. Hohberg, Seismic arch dam analysis with full joint non-linearity, Proceeding of International Conference on Dam Fracture, Denver, Colorado, 1991, 61-75.

6. D. T. Lau and B. Noruziaan et al, Modelling of contraction joint and shear sliding effects on earthquake response of arch dams, Earthquake Engineering and Structural Dynamics 27, 1013-1029, 1998.

7. C. H. Zhang and J. W. Pan et al, Influence of seismic input mechanisms and radiation damping on arch dam response, Soil Dynamics and Earthquake Engineering 29, 1282-1293, 2009.

8. P. A. Cundall and R. D. Hart, Numerical modeling of discontinua, Engineering Computations 9, 101-113, 1992. 